\title{
Protective effects of fucoidan purified from Undaria pinnatifida against UV-irradiated skin photoaging
}

\author{
Rongrong Jing $^{1 \#}$, Keke Guo ${ }^{1 \#}$, Yulan Zhong ${ }^{1}$, Lusheng Wang ${ }^{1}$, Jungang Zhao ${ }^{1}$, Bingyan Gao ${ }^{1}$, Ziyi Ye ${ }^{1}$, \\ Yu Chen ${ }^{1}$, Xuenan $\mathrm{Li}^{1}$, Nuo Xu ${ }^{1}$, Xuan Xuan \\ ${ }^{1}$ College of Life and Environmental Sciences, Wenzhou University, Wenzhou, China; ${ }^{2}$ Department of Dermatology, The First Affiliated Hospital of \\ Wenzhou Medical University, Wenzhou, China \\ Contributions: (I) Conception and design: N Xu, X Xuan; (II) Administrative support: R Jing, K Guo; (III) Provision of study materials or patients: K \\ Guo, J Zhao, Y Chen; (IV) Collection and assembly of data: L Wang, B Gao, Z Ye; (V) Data analysis and interpretation: R Jing, Y Zhong, X Li; (VI) \\ Manuscript writing: All authors; (VII) Final approval of manuscript: All authors. \\ \#These authors contributed equally to this work. \\ Correspondence to: Nuo Xu. College of Life and Environmental Sciences, Wenzhou University, Wenzhou, China. Email: seer.sino@hotmail.com; Xuan \\ Xuan. Department of Dermatology, The First Affiliated Hospital of Wenzhou Medical University, Wenzhou, China. Email: XuanXuanWY@hotmail.com.
}

\begin{abstract}
Background: Exposure to ultraviolet (UV) radiation (UVB and UVA) is the most well-known extrinsic factor that induces skin aging. Fucoidan has been shown to possess antiphotoaging effects against UV irradiation and can be used as an ingredient in the pharmaceutical industry. The present study evaluated the photoprotective effect of fucoidan purified from Undaria pinnatifida (UPF) on UV-induced skin photoaging and explored its potential molecular mechanism.

Methods: To evaluate the effect of UPF on UV-induced skin aging, HaCaT cells and HFF-1 cells were pretreated with or without UPF and then exposed to UVB and UVA radiation, respectively, and the levels of cellular senescence, reactive oxygen species (ROS) production and mitochondrial dysfunction were evaluated. The mitochondrial ROS (mROS) was stained through MitoSOX, and the confocal microscope was used to capture the images. For further exploration of AMPK/SIRT-1/PGC-1 $\alpha$ signaling, western blot was employed.

Results: The results demonstrated that pretreatment of HaCaT and HFF-1 cells with UPF ameliorated cellular senescence, ROS and mROS overproduction, and mitochondrial dysfunction caused by UV exposure. This research also revealed that UPF could activate the AMPK/SIRT-1/PGC-1 $\alpha$ signaling pathway to promote mitochondrial biogenesis.

Conclusions: UPF can ameliorate UV-induced skin photoaging through inhibition of ROS production via the alleviation of mitochondrial dysfunction by regulating the SIRT-1/PGC-1 $\alpha$ signaling pathway.
\end{abstract}

Keywords: Photoaging; Undaria pinnatifida (UPF); ultraviolet (UV); reactive oxygen species (ROS); mitochondrial dysfunction

Submitted Jul 09, 2021. Accepted for publication Jul 22, 2021.

doi: 10.21037/atm-21-3668

View this article at: https://dx.doi.org/10.21037/atm-21-3668

\section{Introduction}

The skin is a barrier that segregates the body from the environment and provides overall protection. As the most visible organ of the body, aging has a perceived negative influence on people's appearance (1). Skin aging is characterized by dryness, wrinkling, sagging, increased laxity, and aberrant pigmentation (2). It has been suggested that skin aging is induced by intrinsic and extrinsic factors. While intrinsic skin aging is due entirely to the passage of time and is influenced by genetics, extrinsic aging can occur earlier as a result of environmental factors including air pollution, smoking, ultraviolet (UV) radiation, stress, and a lack of 
nutrition (3-6). The skin is composed of two layers: the epidermis and dermis. The epidermis is primarily composed of keratinocytes, which undergo continuous renewal and resemble a brick-mortar structure. The dermis is the main tissue in the skin and consists of fibroblasts that produce an extracellular matrix of collagenous and elastic fibers (7). In aging skin, senescent keratinocytes and fibroblasts have been observed in the dermis and epidermis, respectively $(8,9)$. Senescent keratinocytes cause thinning of the epidermis and aggravate skin sensitivity due to proliferation arrest and the secretion of senescence-associated secretory phenotype (SASP) factors. Senescent fibroblasts degrade collagen, elastin, and fibronectin by secreting SASP factors, such as matrix metalloproteinases, causing relaxation and wrinkle formation in aged skin $(10,11)$.

Notably, UV radiation is the primary factor in extrinsic skin aging, known as photoaging, and accounts for approximately $80 \%$ of skin aging. UV can be divided into the following three types based on wavelength: UVA (320$400 \mathrm{~nm})$, UVB (280-320 nm), and UVC (200-280 nm). UVC is blocked from reaching the Earth's surface by oxygen and the atmospheric ozone layer. Ultraviolet B (UVB), which has a shorter wavelength, can only penetrate the epidermis and trigger aging of this layer. In contrast, the longer wavelengths of Ultraviolet A (UVA) penetrate deeply into the dermis and induce the degradation of collagen and elastin, causing the elasticity of the skin and wrinkle formation (12-14). Both UVA and UVB contribute to photoaging. UV irradiation is known to induce the generation of ROS, which induce inflammatory cytokine expression and contribute to the synthesis of a series of matrix metalloproteinases that cause collagen degradation, resulting in wrinkles (15-17).

Seaweed is a marine plant that can protect itself from photodamage, including from UVB irradiation. Fucoidans are a family of sulfated polyfucose polysaccharides extracted from brown seaweeds. These substances possess various pharmacological properties that exert antitumor, immunomodulatory, antioxidant, and anti-inflammatory effects $(18,19)$. Previous studies have demonstrated that fucoidans also have antiphotoaging effects against UV irradiation and can be used as an ingredient in pharmaceuticals (20-22). One previous study examined the UVB-protective effects of a low-molecular-weight fucoidan from Sargassum confusum, which included reducing intracellular ROS levels, DNA damage, inflammatory responses, and apoptosis in human keratinocytes (23). In vivo studies have also shown that fucoidan purified from Saccharina japonica can reduce ROS levels, cell death, and nitric oxide (NO) production in UVB-irradiated zebrafish (18). Fucoidan purified from Undaria pinnatifida (UPF) also exerts its photoprotective effect by augmenting the immune response and decreasing the matrix metalloproteinase-1 (MMP-1) level in UVB-irradiated mouse skin although its mechanism remains unknown (24). Based on results of our previous study (25), we hypothesized that UPF exerts antiphotoaging effects against UV irradiation. This study was aimed to explore the effect of UPF on UV-irradiated keratinocytes and fibroblasts and to explore the potential underlying mechanisms. We present the following article in accordance with the MDAR reporting checklist (available at https://dx.doi.org/10.21037/ atm-21-3668).

\section{Methods}

\section{Cell lines and cell culture}

HaCaT and HFF-1 cell lines were purchased from Zhong Qiao Xin Zhou Biotechnology Co., Ltd. (Shanghai, China). $\mathrm{HaCaT}$ and HFF-1 cells were cultured in Dulbecco's Modified Eagle Medium (DMEM; Gibco, Life Technologies Corporation, NY, USA) supplemented with $10 \%$ fetal bovine serum (FBS; Gibco, Life Technologies Corporation) in a humidified atmosphere with $5 \% \mathrm{CO}_{2}$ at $37^{\circ} \mathrm{C}$.

\section{UV irradiation}

$\mathrm{HaCaT}$ cells were incubated with UPF $(0,50$, and $200 \mu \mathrm{g} / \mathrm{mL}$ ) for 4 hours. After this, HaCaT cells were exposed to UVB $\left(20 \mathrm{~mJ} / \mathrm{cm}^{2}\right)$, while HFF-1 cells were incubated with UPF $(0,50$, and $200 \mu \mathrm{g} / \mathrm{mL})$ for 4 hours. The cells were then exposed to UVA $\left(5 \mathrm{~J} / \mathrm{cm}^{2}\right)$ and incubated with DMEM for different durations.

\section{Quantitative real-time polymerase chain reaction}

$\mathrm{HaCaT}$ and HFF-1 cells were treated with UPF for 4 hours and then exposed to UVB or UVA, respectively, which was followed by 6 hours of incubation. RNA was isolated from cells using an RNA extraction kit (Biomiga, San Diego, CA, USA), and $1 \mu \mathrm{g}$ of total RNA was reverse transcribed with a PrimeScript RT Reagent Kit (Takara, Dalian, China). Quantitative real-time polymerase chain reaction (qRTPCR) was performed using SYBR Green Master Mix (Applied Biosystems, Foster City, CA, USA). The primer 
Table 1 Primer sequences for quantitative qRT-PCR

\begin{tabular}{lll}
\hline Gene & Primer & Primer sequence $\left(5^{\prime}-3^{\prime}\right)$ \\
\hline IL-6 & Forward & CCGAAGGACGGGAGCAG \\
& Reverse & GGGTCAGGGGTGGTTATTGC \\
IL-8 & Forward & AGCAGTTCCACAGGCACA \\
& Reverse & ACTCTGGTTGGCTTCCTTCA \\
MMP-1 & Forward & TGGGCTGAAAGTGACTGGGAAAC \\
& Reverse & ACATCTGGGCTGCTTCATCACC \\
MMP-3 & Forward & AGTTCCTTGGATTGGAGGTGACG \\
& Reverse & TTCGGGATGCCAGGAAAGGTTC
\end{tabular}

IL-6, interleukin-6; IL-8, interleukin-8; MMP-1, matrix metalloproteinase-1; MMP-3, matrix metalloproteinase-3.

sequences are shown in Table 1. The expression of each gene relative to $\beta$-actin was calculated using the $2^{-\Delta \Delta \mathrm{Ct}}$ method.

\section{Senescence-associated B-galactosidase staining}

$\mathrm{HaCaT}$ and HFF-1 cells were fixed in cold $4 \%$ paraformaldehyde for 3 minutes, which was followed by incubation with $200 \mu \mathrm{L}$ of SPiDER- $\beta$ Gal (Dojindo Laboratories, Tokyo, Japan) for 15 minutes. The fluorescence of senescence-associated $\beta$-galactosidase (SA$\beta$-gal)-positive cells were captured using a fluorescence microscope (Nikon, Tokyo, Japan).

\section{Intracellular ROS measurement}

The UV-induced overproduction of ROS in $\mathrm{HaCaT}$ and HFF-1 cells was determined using a ROS assay kit (Beyotime, China) according to the manufacturer's protocol. Briefly, the cells were washed twice with phosphatebuffered saline (PBS) and then stained with dichlorodihydro-fluorescein diacetate (DCFH-DA; $5 \mu \mathrm{M}$ ) for 15 minutes at $37{ }^{\circ} \mathrm{C}$ in a $5 \% \mathrm{CO}_{2}$ incubator. Subsequently, the ROS production was detected by ACEA NovoCyte flow cytometer (Agilent, Santa Clara, CA, USA).

\section{Mitochondrial ROS measurement}

$\mathrm{HaCa}$ T cells and HFF-1 cells were treated with UPF for 4 hours, irradiated with UVB or UVA, and incubated postirradiation for 2 hours. After that, the cells were stained with MitoSOX (Invitrogen, Waltham, MA, USA) for 30 minutes. The cell nuclei were stained with 40,6-diamidino-2- phenylindole (DAPI), and images were captured using a confocal microscope (Nikon, Tokyo, Japan).

\section{FC-1 staining}

The MMP loss was evaluated by JC-1 fluorescent probes (Beyotime Institute of Biotechnology, Jiangsu, China). First, UPF pretreated $\mathrm{HaCaT}$ cells and HFF-1 cells were incubated post-irradiation for 1 hour. Subsequently, the cells were stained with $5 \mu$ M JC- 1 for 20 minutes. Finally, a fluorescence microscope was used to detect the MMP loss of cells.

\section{Mitochondrial mass analysis}

$\mathrm{HaCaT}$ cells and HFF-1 cells were treated in the same manner as that described for JC-1 staining. The cells were first incubated with $50 \mathrm{nM}$ Mito Tracker Green (Beyotime, Jiangsu, China) in DMEM for 1 hour at $37^{\circ} \mathrm{C}$. Confocal microscopy was employed to detect the fluorescence of the mitochondria.

\section{Western blot}

HaCaT Cells and HFF-1 cells were pretreated with UPF for 4 hours, exposed to UVB or UVA irradiation, respectively, and incubated post-irradiation for 30 minutes. After this, total protein was extracted from cells. Then, $20 \mu \mathrm{g}$ protein was subjected to $10 \%$ SDS-PAGE and transferred to a polyvinylidene fluoride (PVDF) membrane. The membrane was immunolabeled with antibodies for AMPK, phospho-AMPK, PGC-1 $\alpha$, SIRT-1, and tubulin (Cell Signaling Technology, Beverly, MA, USA) and followed by further incubation with goat anti-mouse or goat anti-rabbit secondary antibodies (Cell Signaling Technology). The immune complexes were finally detected with a chemiluminescence substrate (Thermo Scientific, USA). Detection was performed with chemiluminescence substrate (Thermo Scientific).

\section{Statistical analysis}

All data were analyzed by GraphPad Prism 5.01 (GraphPad, San Diego, CA, USA) and are expressed as the mean \pm standard deviation (SD) derived from 3 independent experiments. A comparison of data between groups was performed using one-way analysis of variance (ANOVA) and followed by Dunnett's test. P values $<0.05$ or $<0.01$ 
were considered statistically significant.

\section{Results}

Protective effects of UPF against UVB-irradiated $\mathrm{HaCaT}$ cell senescence and UVA-irradiated HFF-1 cell senescence

Cellular senescence is the main effector in photoaging, and senescent cells accumulate SA- $\beta$-gal, which distinguishes them from quiescent cells (26). HaCaT cells exposed to $20 \mathrm{~mJ} / \mathrm{cm}^{2}$ dose of UVB followed by 12 hours of incubation showed a significant increase in the number of SA- $\beta$-galpositive $\mathrm{HaCaT}$ cells (senescent $\mathrm{HaCaT}$ cells) compared with non-irradiated $\mathrm{HaCaT}$ cells (control); however, the number of UVB-induced senescent $\mathrm{HaCaT}$ cells was reduced significantly when cells were pretreated with UPF (20 and $50 \mu \mathrm{g} / \mathrm{mL}$ ) before UVB irradiation (Figure 1A). The messenger RNA (mRNA) expression of interleukin 6 (IL-6) and IL-8 were also elevated after UVB exposure, but pretreatment of UPF (20 and $50 \mu \mathrm{g} / \mathrm{mL}$ ) suppressed the levels of IL-6 and IL-8 (Figure 1B). Thus, the results shown in Figure $1 A$ and $B$ indicate that UPF could prevent $\mathrm{HaCaT}$ cells from UVB-induced senescence. HFF-1 cells irradiated with $5 \mathrm{~J} / \mathrm{cm}^{2}$ UVA followed by 12 hours of culture showed a significant increase in the number of senescent HFF-1 cells, whereas UPF $(50 \mu \mathrm{g} / \mathrm{mL})$ significantly reduced UVAinduced senescence of HFF-1 cells (Figure 1C). Under these conditions, the mRNA expression of MMP-1 and MMP-3 were significantly inhibited when the HFF-1 cells were treated with UPF (20 and $50 \mu \mathrm{g} / \mathrm{mL}$ ) before UVA irradiation (Figure 1D). The results shown in Figure 1C and $\mathrm{D}$ indicate that UPF could prevent HFF-1 cells from UVAinduced senescence. Taken together, these observations reveal the protective effects of UPF against $\mathrm{UV}$-induced photoaging.

\section{UPF attenuated ROS production caused by UV exposure in HaCaT and HFF-1 cells}

Photoaging is also associated with the generation and accumulation of ROS in skin cells (27). In this study, the effect of UPF on the UV-induced production of ROS was demonstrated using flow cytometry. The results in Figure $2 \mathrm{~A}$ indicate that UVB can cause an increase in ROS in HaCaT cells, whereas UPF $(50 \mu \mathrm{g} / \mathrm{mL})$ can significantly inhibit the UVB-induced production of ROS. The pretreatment of HFF-1 cells with UPF (20 and $50 \mu \mathrm{g} / \mathrm{mL}$ ) also significantly decreased the generation of ROS triggered by UVA irradiation (Figure 2B). These results suggest that UPF is capable of reducing UV-induced ROS production.

\section{UPF ameliorated the extent of mROS caused by UV irradiation in HaCaT and HFF-1 cells}

One of the major sources of ROS is mitochondria [referred to as mitochondrial ROS (mROS)], which are instrumental for the initiation and maintenance of cellular senescence $(28,29)$. mROS are generated as byproducts of the electron transport chain (ETC) in the mitochondrial matrix or intermembrane space. In this study, MitoSOX was employed to determine the levels of mROS, and the effect of UPF on mROS generation was demonstrated. In $\mathrm{HaCaT}$ cells subjected to UVB radiation, a significant increase in the amount of mROS was detected when compared to the untreated control cells. This effect was efficiently counteracted by pretreatment with UPF (20 and $50 \mu \mathrm{g} / \mathrm{mL}$; Figure 3A). The results in Figure $3 B$ demonstrate that UVA irradiation led to a significant elevation in mROS levels compared to the untreated HFF-1 cells and that UPF $(50 \mu \mathrm{g} / \mathrm{mL})$ reduced the UVA-induced production of mROS. These data are in line with the results that demonstrated the overproduction of ROS induced by UV radiation to be inhibited by pretreatment with UPF.

\section{UPF ameliorated mitochondrial dysfunction caused by UV irradiation in HaCaT and HFF-1 cells}

During the aging process, mitochondria lose their function, leading to a decline in mitochondrial biogenesis and adenosine trisphosphate production (ATP) production, which halts the activities of enzymes in the ETC and results in the generation of ROS (30). To determine the effect of UPF on mitochondrial function, the MMP and mitochondrial mass of $\mathrm{HaCaT}$ and HFF-1 cells were detected following UV exposure. Compared with the nonirradiated control sample, the mitochondrial mass of $\mathrm{HaCaT}$ and HFF-1 cells was significantly reduced after UVB and UVA irradiation, respectively. The mitochondrial mass was significantly increased when the cells were treated with UPF (20 and $50 \mu \mathrm{g} / \mathrm{mL}$ ) before UVB or UVA irradiation (Figure 4A,B). With UV irradiation, the HaCaT and HFF-1 cells showed a reduction in MMP, whereas pretreatment with UPF resulted in an elevated level of MMP (Figure 4C,D). Thus, UPF can prevent mitochondrial dysfunction in $\mathrm{HaCaT}$ and HFF-1 cells. 

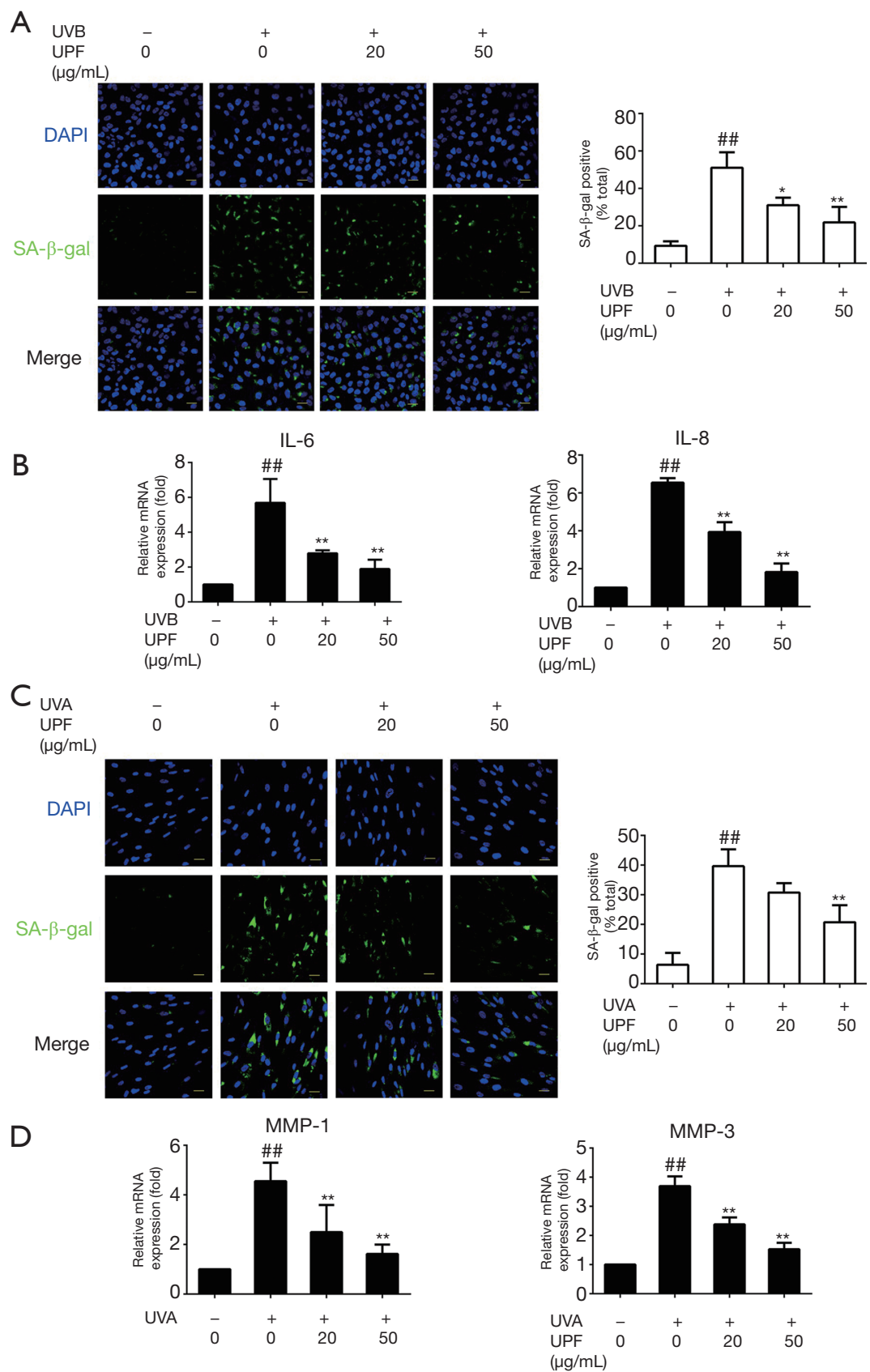

Figure 1 Effect of UPF on UV-induced HaCaT cells and HFF-1 cells senescence. (A) Fluorescence staining of SA- $\beta$-gal-positive HaCaT cells was quantified after incubation for 12 hours following UVB irradiation. Scale bar $=20 \mu m$. (B) The mRNA expression of IL-6 and IL-8 in HaCaT cells after 6-hour incubation following UVB irradiation. (C) Fluorescence staining of senescent HFF-1 cells was quantified after 12-hour incubation following UVA irradiation. Scale bar $=20 \mu \mathrm{m}$. (D) The mRNA expression of MMP-1 and MMP-3 in HFF-1 cells after 6-hour incubation following UVA irradiation. All data are expressed as the mean \pm SD of 3 independent experiments; ${ }^{\# \prime} \mathrm{P}<0.01$ versus non-irradiated group; while ${ }^{*} \mathrm{P}<0.05$ and ${ }^{* *} \mathrm{P}<0.01$ versus $\mathrm{UV}$ group. UPF, Undaria pinnatifida; UV, ultraviolet; UVB, ultraviolet B; UVA, ultraviolet A; IL-6, interleukin-6; IL-8, interleukin-8; MMP-1, matrix metalloproteinase-1; MMP-3, matrix metalloproteinase-3; SD, standard deviation. 

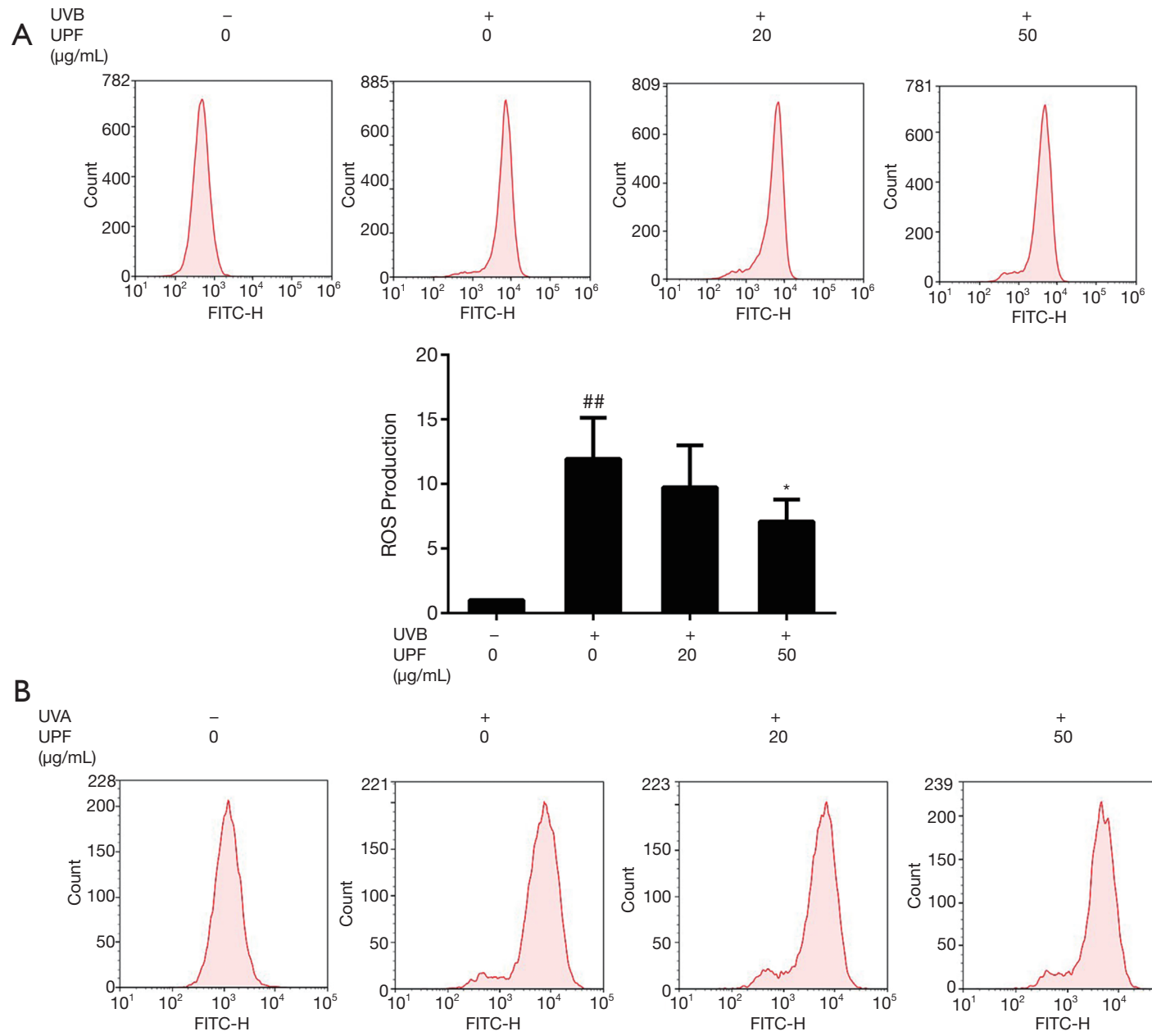

$(\mu \mathrm{g} / \mathrm{mL})$

$\stackrel{+}{20}$

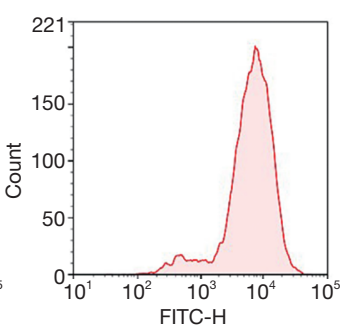

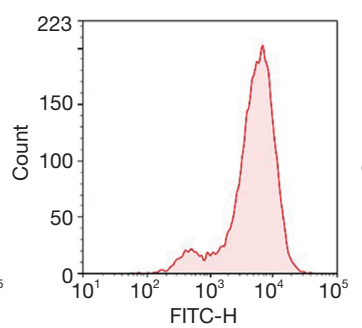

$\stackrel{+}{50}$
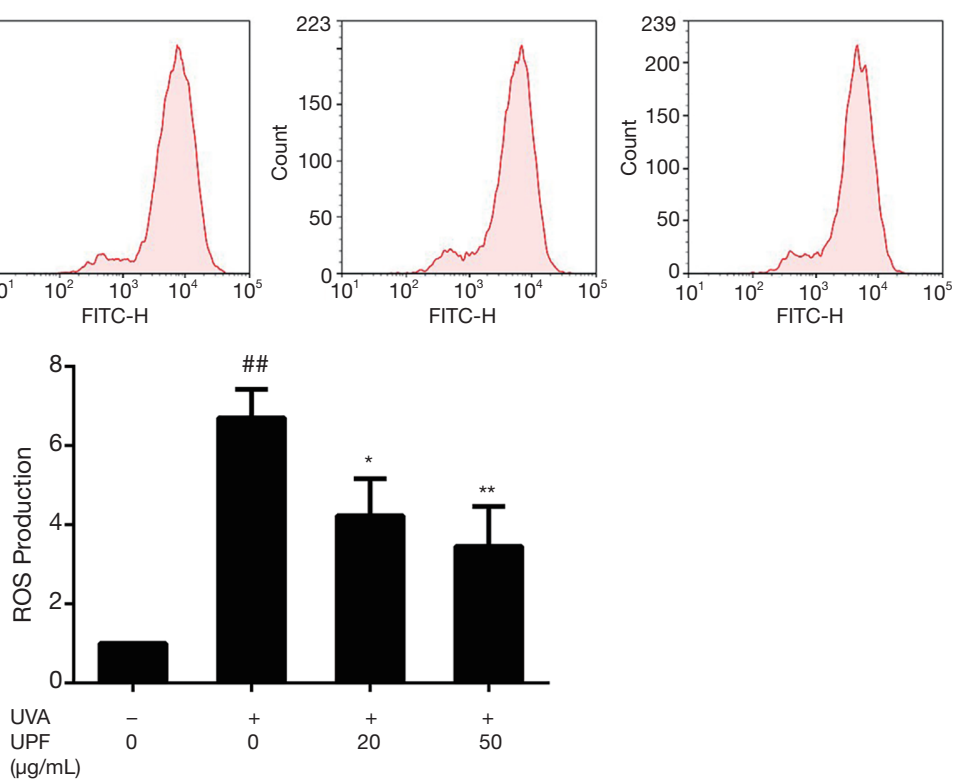

Figure 2 Effect of UPF on the ROS production induced by UV irradiation. (A) Intracellular ROS generation in $\mathrm{HaCaT}$ cells was detected and quantified by flow cytometry after 4-hour incubation following UVB irradiation. (B) Intracellular ROS generation in HFF-1 cells was detected and quantified by flow cytometry after 4-hour incubation following UVA irradiation. All data are expressed as the mean \pm SD of 3 independent experiments; ${ }^{\# \prime} \mathrm{P}<0.01$ versus non-irradiated group; ${ }^{*} \mathrm{P}<0.05$ and ${ }^{* *} \mathrm{P}<0.01$ versus $\mathrm{UV}$ group. UPF, Undaria pinnatifida; ROS, reactive oxygen species; UV, ultraviolet; UVB, ultraviolet B; UVA, ultraviolet A; SD, standard deviation. 


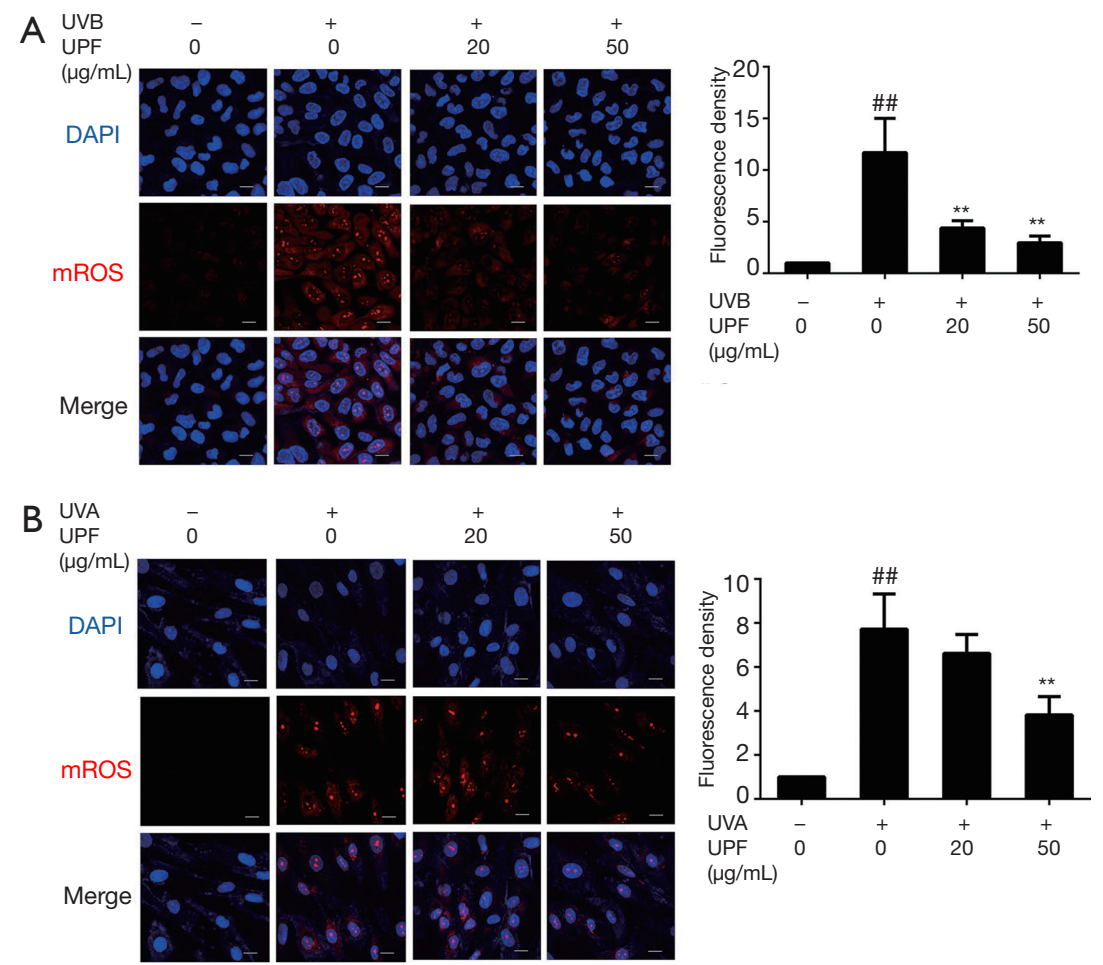

Figure 3 Effect of UPF on UV irradiation-induced mROS overproduction. (A) Fluorescence staining of mROS in HaCaT cells was quantified after incubation for 2 hours following UVB irradiation. (B) Fluorescence staining of mROS in HFF-1 cells was quantified after incubation for 2 hours following UVA irradiation. Scale bar $=20 \mu \mathrm{m}$. All data are expressed as the mean \pm SD of 3 independent experiments; ${ }^{\# \#} \mathrm{P}<0.01$ versus non-irradiated group; ${ }^{* *} \mathrm{P}<0.01$ versus $\mathrm{UV}$ group. UPF, Undaria pinnatifida; mROS, mitochondrial reactive oxygen species; UV, ultraviolet; UVB, ultraviolet B; UVA, ultraviolet A; SD, standard deviation.

\section{UPF activated the AMPK/ SIRT-1/ PGC-1a signaling patbway}

It is known that AMPK and SIRT-1 promote mitochondrial biogenesis and oxidative capacity by regulating PGC-1 $\alpha$ and preventing mitochondrial dysfunction $(31,32)$. In the present study, the cultured $\mathrm{HaCaT}$ and HFF-1 cells were treated with UPF (20 and $50 \mu \mathrm{g} / \mathrm{mL}$ ) prior to UVB or UVA irradiation, respectively. UVB irradiation decreased the phosphorylation of AMPK and expression of SIRT-1 and PGC- $1 \alpha$ as compared to the nonirradiated control cells. Furthermore, treatment with UPF stimulated AMPK activity and increased the expression of SIRT- 1 and PGC- $1 \alpha$ in the HaCaT cells (Figure 5A). Furthermore, treatment with UPF increased the phosphorylation of AMPK, and the expression of SIRT-1 and PGC- $1 \alpha$ in the HFF-1 cells (Figure $5 B$ ). These data indicate that UPF could activate the AMPK/SIRT-1/PGC-1 $\alpha$ signaling pathway to promote mitochondrial biogenesis.

\section{Discussion}

Aging is a biological process that occurs in every individual from the moment of birth, and, as the most voluminous organ of the body, the skin shows visible signs of aging as one becomes older (33). The dramatic increase in the aging population and the psychosocial impact of aged skin has created a demand for preventing or reversing skin aging (34). UV radiation in sunlight is the most well-known extrinsic factor that induces skin aging. Fucoidans have been demonstrated to exert antiphotoaging effects against UV irradiation. We found that the fucoidan from UPF can ameliorate epidermal barrier disruption (25). In the present study, we explored the effect of UPF on UV-induced epidermis and dermis aging. Our results indicate that UPF could inhibit ROS production by alleviating mitochondrial dysfunction and reducing the number of senescent $\mathrm{HaCaT}$ and HFF-1 cells.

In aged skin, senescent keratinocytes have been 


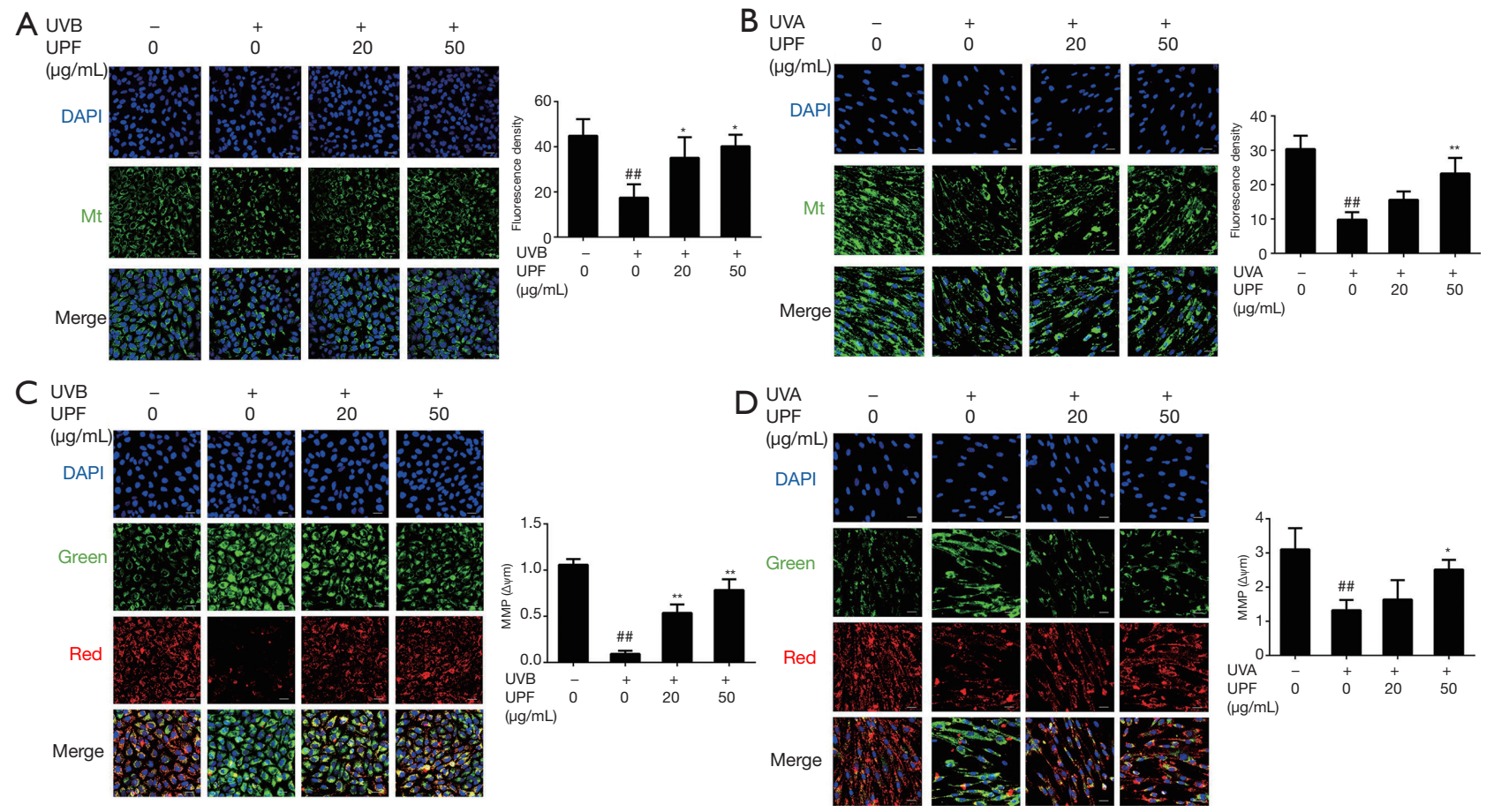

Figure 4 Effect of UPF on the mitochondrial dysfunction caused by UV irradiation. (A) Fluorescence staining of mitochondrial mass in $\mathrm{HaCaT}$ cells was quantified after incubation for 2 hours following UVB irradiation. (B) Fluorescence staining of mitochondrial mass in HFF-1 cells was quantified after incubation for 2 hours following UVA irradiation. (C) Fluorescence staining of MMP in HaCaT cells was quantified after incubation for 2 hours following UVB irradiation. (D) Fluorescence staining of MMP in HFF-1 cells was obtained and quantified after incubation for 2 hours following UVA irradiation. Scale bar $=20 \mu \mathrm{m}$. All data are expressed as the mean \pm SD of 3 independent experiments; ${ }^{\# \#} \mathrm{P}<0.01$ versus non-irradiated group; ${ }^{*} \mathrm{P}<0.05$ and ${ }^{* *} \mathrm{P}<0.01$ versus the UV group. UPF, Undaria pinnatifida; UV, ultraviolet; UVB, ultraviolet B; UVA, ultraviolet A; MMP, mitochondrial membrane potential; SD, standard deviation.

observed in the epidermis and senescent fibroblasts in the epidermis (9). Keratinocytes are the major cell type of the epidermis and provide an efficient and continuously renewing barrier to the environment (35). UV irradiation, by impairing the cellular redox status, can lead to the excessive generation of ROS, which are the main contributors to skin aging (15). In our observations, $\mathrm{HaCa}$ T cells exposed to UVB exhibited elevated ROS production and an increase in the number of senescent $\mathrm{HaCaT}$ cells. This was accompanied by the secretion of SASPs, such as IL-6 and IL-8, which play a critical role in immunosuppression. However, treatment of HaCaT cells with UPF before UVB irradiation reduced the production of ROS, resulting in marked protection against UVB-induced $\mathrm{HaCaT}$ cells senescence. Fibroblasts are the most abundant and most studied senescence-related cell type of the dermis. Once these cells are exposed to UVA irradiation, elevated ROS activate signaling cascades that promote the synthesis of matrix metalloproteinases, which are important contributors to the degradation of collagen and other extracellular matrix components in the dermis, resulting in skin wrinkling and loss of elasticity (36-38). We found that HFF-1 cells irradiated with UVA exhibited ROS overproduction and increased expression of MMP-1 and MMP-3, leading to senescence. These effects can be suppressed by UPF, providing preliminary evidence that UPF may be crucial for antiphotoaging.

Mitochondria also play a critical role in aging. Mitochondria are the major source of ROS, and overproduction of mROS cause DNA damage and activation of CDK1N1 (p21). Subsequently, p21 induces mitochondrial dysfunction and permanent cell cycle arrest, which is instrumental in the initiation and maintenance of cellular senescence $(29,39,40)$. Mitochondrial dysfunction has been considered a driver of aging $(30,41)$. mROS-derived mitochondrial DNA damage results in decreased MMP and mitochondrial 

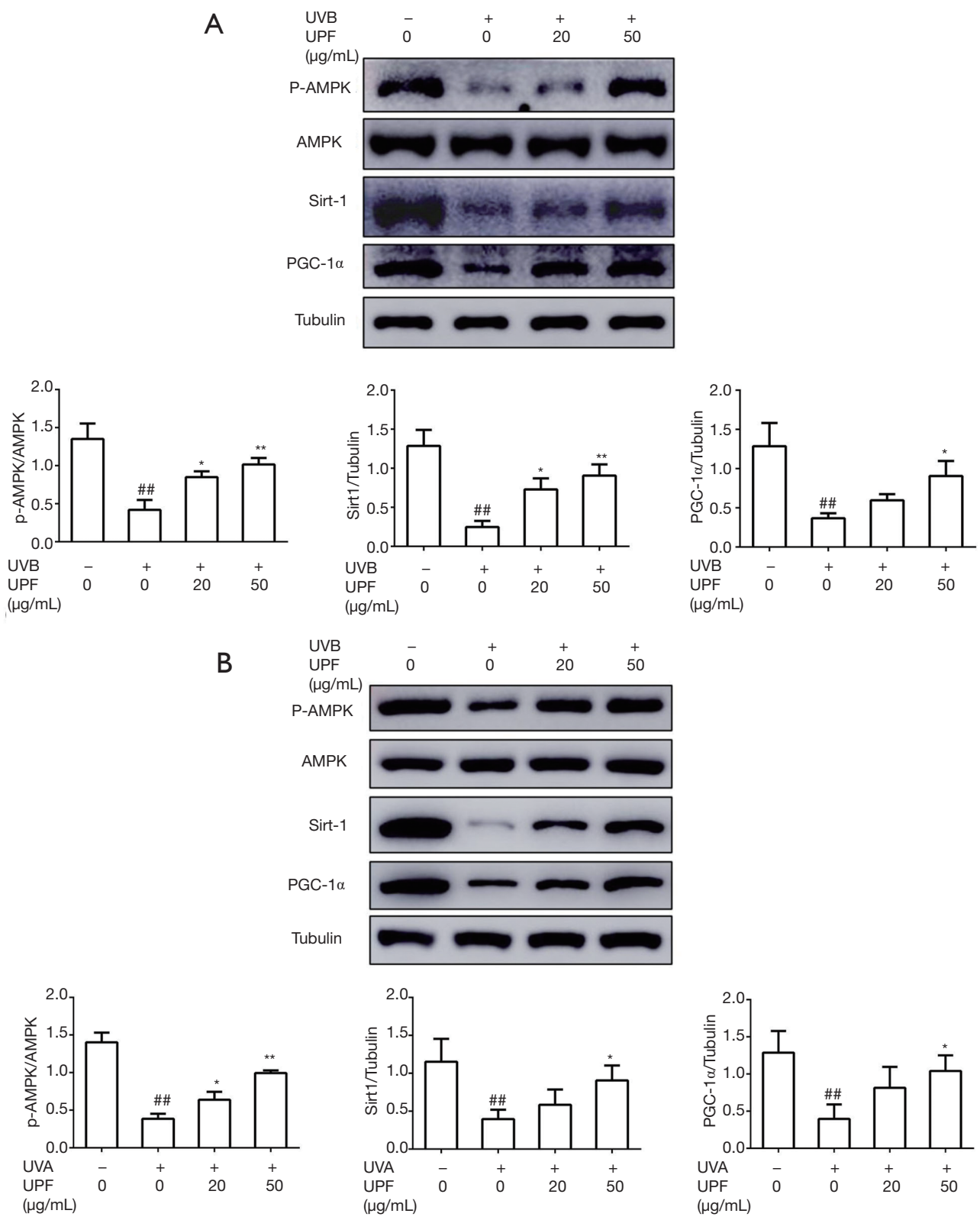

Figure 5 Effect of UPF on the AMPK/SIRT-1/PGC-1 $\alpha$ signaling pathway following UV irradiation. (A) HaCaT cells were pretreated with UPF for 4 hours, exposed to UVB, and incubated for a further 30 minutes; the protein levels of phospho-AMPK, AMPK, SIRT-1, and PGC-1 $\alpha$ were assessed by western blot. The graphs show a quantitative analysis of protein expression. (B) HFF-1 cells were pretreated with UPF for 4 hours, exposed to UVA, and incubated for a further 30 minutes; the protein levels of phospho-AMPK, AMPK, SIRT-1, and PGC- $1 \alpha$ were assessed by western blot. The graphs show a quantitative analysis of protein expression. All data are expressed as the mean $\pm \mathrm{SD}$ of 3 independent experiments; ${ }^{* \#} \mathrm{P}<0.01$ versus non-irradiated group; ${ }^{*} \mathrm{P}<0.05$ and ${ }^{* *} \mathrm{P}<0.01$ versus the $\mathrm{UV}$ group. UPF, Undaria pinnatifida; mROS, mitochondrial reactive oxygen species; UV, ultraviolet; UVB, ultraviolet B; UVA, ultraviolet A; phospho-AMPK, phosphorylated AMP-activated protein kinase; AMPK, AMP-activated protein kinase; SIRT-1, silent information regulator-1; PGC-1 $\alpha$, peroxisome proliferator-activated receptor $\gamma$ coactivator $1 \alpha$; SD, standard deviation. 
biogenesis (42). In addition, mitochondrial dysfunction leads to ROS production, which further disrupts mitochondrial and cellular homeostasis (43). In this study, we found that UPF reduced the elevation of mROS and attenuated the reduction of mitochondrial mass and the loss of MMP induced by UV irradiation, which may explain the inhibitory effect of UPF on the generation of ROS induced by UV irradiation.

With aging, mitochondrial biogenesis is reduced. Mitochondrial biogenesis can produce new and healthy mitochondria and is dependent on different signaling cascades (42). In general, PGC- $1 \alpha$ is thought to act as a central hub in mitochondrial biogenesis by interacting with downstream factors including peroxisome proliferatoractivated receptors (PPAR $\beta$ ), nuclear respiratory factors (NRFs), estrogen-related receptors (ERRs), and mitochondrial transcription factor A (Tfam). This in turn activates the expression of nuclear and mitochondrial genes that encode mitochondrial proteins $(44,45)$. A decline in mitochondrial biogenesis may result from an age-dependent reduction in PGC-1 $\alpha$ (46), and the overexpression of PGC-1 $\alpha$ in skeletal muscles has been found to be associated with an improvement in mitochondrial function in aged mice (47). AMPK and SIRT-1 could promote mitochondrial biogenesis by regulating PGC- $1 \alpha$ activity via phosphorylation and deacetylation, respectively $(31,32,48)$. AMPK deficiency can lead to age-associated ROS generation; meanwhile, SIRT-1-deficient primary myoblasts can decrease mitochondrial DNA content and MMP, and SIRT-1 deletion promotes the production of mROS and alters mitochondrial properties (49). We found that $\mathrm{HaCaT}$ cells irradiated with UVB showed decreased phosphorylation of AMPK and significant downregulation of SIRT-1 and PGC- $1 \alpha$ compared to nonirradiated cells. However, the HaCaT cells displayed a marked increase in AMPK phosphorylation and SIRT-1 and PGC- $1 \alpha$ expression when pretreated with UPF. In agreement with the results of $\mathrm{HaCaT}$ cells, UPF also significantly elevated the expression of SIRT-1 and PGC- $1 \alpha$. These data suggest that the effects of UPF in promoting mitochondrial biogenesis and the amelioration of mitochondrial dysfunction are related to the activation of the AMPK/SIRT-1/PGC- $1 \alpha$ signaling pathway. Consequently, these results demonstrate, for the first time, that UPF may protect UVB-irradiated keratinocytes and UVA-irradiated fibroblasts from ROS overproduction by attenuating mitochondrial dysfunction and inhibiting UVinduced photoaging. However, the precise mechanism of the observed UPF effect requires further investigation.

\section{Conclusions}

In summary, we have demonstrated that UPF can attenuate $\mathrm{UV}$-induced photoaging by protecting UVB-irradiated $\mathrm{HaCaT}$ cells and UVA-irradiated HFF-1 cells from ROS overproduction via the alleviation of mitochondrial dysfunction. Our observations show that UPF can stimulate the activation of the AMPK/SIRT-1/PGC- $1 \alpha$ pathway to increase mitochondrial biogenesis in $\mathrm{HaCaT}$ cells and HFF-1 cells. These findings suggest that UPF has great potential in the application of antiphotoaging.

\section{Acknowledgments}

We thank Dr. Alan K. Chang (Wenzhou University) for his valuable work in revising the language of the manuscript. Funding: This research was funded by National Natural Science Foundation of China (No. 81903736).

\section{Footnote}

Reporting Checklist: The authors have completed the MDAR reporting checklist. Available at https://dx.doi. org/10.21037/atm-21-3668

Data Sharing Statement: Available at https://dx.doi. org/10.21037/atm-21-3668

Conflicts of Interest: All authors have completed the ICMJE uniform disclosure form (available at https://dx.doi. org/10.21037/atm-21-3668). The authors have no conflicts of interest to declare.

Ethical Statement: The authors are accountable for all aspects of the work in ensuring that questions related to the accuracy or integrity of any part of the work are appropriately investigated and resolved.

Open Access Statement: This is an Open Access article distributed in accordance with the Creative Commons Attribution-NonCommercial-NoDerivs 4.0 International License (CC BY-NC-ND 4.0), which permits the noncommercial replication and distribution of the article with the strict proviso that no changes or edits are made and the original work is properly cited (including links to both the 
formal publication through the relevant DOI and the license). See: https://creativecommons.org/licenses/by-nc-nd/4.0/.

\section{References}

1. Zhang S, Duan E. Fighting against Skin Aging: The Way from Bench to Bedside. Cell Transplant 2018;27:729-38.

2. Wang AS, Dreesen O. Biomarkers of Cellular Senescence and Skin Aging. Front Genet 2018;9:247.

3. Friedman O. Changes associated with the aging face. Facial Plast Surg Clin North Am 2005;13:371-80.

4. Kammeyer A, Luiten RM. Oxidation events and skin aging. Ageing Res Rev 2015;21:16-29.

5. Farage MA, Miller KW, Elsner P, et al. Intrinsic and extrinsic factors in skin ageing: a review. Int J Cosmet Sci 2008;30:87-95.

6. Baumann L. Skin ageing and its treatment. J Pathol 2007;211:241-51.

7. Wong R, Geyer S, Weninger W, et al. The dynamic anatomy and patterning of skin. Exp Dermatol 2016;25:92-8.

8. Shuster S, Black MM, McVitie E. The influence of age and sex on skin thickness, skin collagen and density. Br J Dermatol 1975;93:639-43.

9. Velarde MC, Demaria M. Targeting Senescent Cells: Possible Implications for Delaying Skin Aging: A MiniReview. Gerontology 2016;62:513-8.

10. Baumann L. How to Use Oral and Topical Cosmeceuticals to Prevent and Treat Skin Aging. Facial Plast Surg Clin North Am 2018;26:407-13.

11. Tobin DJ. Introduction to skin aging. J Tissue Viability 2017;26:37-46.

12. D'Orazio J, Jarrett S, Amaro-Ortiz A, et al. UV radiation and the skin. Int J Mol Sci 2013;14:12222-48.

13. Poon F, Kang S, Chien AL. Mechanisms and treatments of photoaging. Photodermatol Photoimmunol Photomed 2015;31:65-74.

14. Chung JH, Eun HC. Angiogenesis in skin aging and photoaging. J Dermatol 2007;34:593-600.

15. McCullough JL, Kelly KM. Prevention and treatment of skin aging. Ann N Y Acad Sci 2006;1067:323-31.

16. Bai B, Liu Y, You Y, et al. Intraperitoneally administered biliverdin protects against UVB-induced skin photodamage in hairless mice. J Photochem Photobiol B 2015;144:35-41.

17. Abbas H, Kamel R, El-Sayed N. Dermal anti-oxidant, anti-inflammatory and anti-aging effects of Compritol ATO-based Resveratrol colloidal carriers prepared using mixed surfactants. Int J Pharm 2018;541:37-47.

18. Su W, Wang L, Fu X, et al. Protective Effect of a FucoseRich Fucoidan Isolated from Saccharina japonica against Ultraviolet B-Induced Photodamage In Vitro in Human Keratinocytes and In Vivo in Zebrafish. Mar Drugs 2020;18:316.

19. Fitton HJ, Stringer DS, Park AY, et al. Therapies from Fucoidan: New Developments. Mar Drugs 2019;17:571.

20. Moon HJ, Park KS, Ku MJ, et al. Effect of Costaria costata fucoidan on expression of matrix metalloproteinase-1 promoter, mRNA, and protein. J Nat Prod 2009;72:1731-4.

21. Wang L, Oh JY, Kim YS, et al. Anti-Photoaging and AntiMelanogenesis Effects of Fucoidan Isolated from Hizikia fusiforme and Its Underlying Mechanisms. Mar Drugs 2020;18:427.

22. Moon HJ, Lee SH, Ku MJ, et al. Fucoidan inhibits UVBinduced MMP-1 promoter expression and down regulation of type I procollagen synthesis in human skin fibroblasts. Eur J Dermatol 2009;19:129-34.

23. Fernando IPS, Dias MKHM, Madusanka DMD, et al. Fucoidan refined by Sargassum confusum indicate protective effects suppressing photo-oxidative stress and skin barrier perturbation in UVB-induced human keratinocytes. Int J Biol Macromol 2020;164:149-61.

24. Maruyama H, Tamauchi H, Kawakami F, et al. Suppressive Effect of Dietary Fucoidan on Proinflammatory Immune Response and MMP-1 Expression in UVB-Irradiated Mouse Skin. Planta Med 2015;81:1370-4.

25. Chen Y, Li X, Gan X, et al. Fucoidan from Undaria pinnatifida Ameliorates Epidermal Barrier Disruption via Keratinocyte Differentiation and CaSR Level Regulation. Mar Drugs 2019;17:660.

26. Campisi J, d'Adda di Fagagna F. Cellular senescence: when bad things happen to good cells. Nat Rev Mol Cell Biol 2007;8:729-40.

27. Petruk G, Del Giudice R, Rigano MM, et al. Antioxidants from Plants Protect against Skin Photoaging. Oxid Med Cell Longev 2018;2018:1454936.

28. Murphy MP. How mitochondria produce reactive oxygen species. Biochem J 2009;417:1-13.

29. Correia-Melo C, Marques FD, Anderson R, et al. Mitochondria are required for pro-ageing features of the senescent phenotype. EMBO J 2016;35:724-42.

30. Trifunovic A, Larsson NG. Mitochondrial dysfunction as a cause of ageing. J Intern Med 2008;263:167-78.

31. Lagouge M, Argmann C, Gerhart-Hines Z, et al. Resveratrol improves mitochondrial function and protects 
against metabolic disease by activating SIRT1 and PGC1alpha. Cell 2006;127:1109-22.

32. Sun C, Zhang F, Ge X, et al. SIRT1 improves insulin sensitivity under insulin-resistant conditions by repressing PTP1B. Cell Metab 2007;6:307-19.

33. Cho BA, Yoo SK, Seo JS. Signatures of photo-aging and intrinsic aging in skin were revealed by transcriptome network analysis. Aging (Albany NY) 2018;10:1609-26.

34. Kazanci A, Kurus M, Atasever A. Analyses of changes on skin by aging. Skin Res Technol 2017;23:48-60.

35. Toutfaire M, Bauwens E, Debacq-Chainiaux F. The impact of cellular senescence in skin ageing: A notion of mosaic and therapeutic strategies. Biochem Pharmacol 2017;142:1-12.

36. Fisher GJ, Kang S, Varani J, et al. Mechanisms of photoaging and chronological skin aging. Arch Dermatol 2002;138:1462-70.

37. Chung JH, Seo JY, Choi HR, et al. Modulation of skin collagen metabolism in aged and photoaged human skin in vivo. J Invest Dermatol 2001;117:1218-24.

38. Pittayapruek P, Meephansan J, Prapapan O, et al. Role of Matrix Metalloproteinases in Photoaging and Photocarcinogenesis. Int J Mol Sci 2016;17:868.

39. Ballinger SW, Patterson C, Yan CN, et al. Hydrogen peroxide- and peroxynitrite-induced mitochondrial DNA damage and dysfunction in vascular endothelial and smooth muscle cells. Circ Res 2000;86:960-6.

40. Nelson G, Wordsworth J, Wang C, et al. A senescent cell bystander effect: senescence-induced senescence. Aging Cell 2012;11:345-9.

41. López-Otín C, Blasco MA, Partridge L, et al. The hallmarks of aging. Cell 2013;153:1194-217.

Cite this article as: Jing R, Guo K, Zhong Y, Wang L, Zhao J, Gao B, Ye Z, Chen Y, Li X, Xu N, Xuan X. Protective effects of fucoidan purified from Undaria pinnatifida against UVirradiated skin photoaging. Ann Transl Med 2021;9(14):1185. doi: $10.21037 / \mathrm{atm}-21-3668$
42. Chistiakov DA, Sobenin IA, Revin VV, et al. Mitochondrial aging and age-related dysfunction of mitochondria. Biomed Res Int 2014;2014:238463.

43. Tahrir FG, Langford D, Amini S, et al. Mitochondrial quality control in cardiac cells: Mechanisms and role in cardiac cell injury and disease. J Cell Physiol 2019;234:8122-33.

44. Peng K, Yang L, Wang J, et al. The Interaction of Mitochondrial Biogenesis and Fission/Fusion Mediated by PGC-1 $\alpha$ Regulates Rotenone-Induced Dopaminergic Neurotoxicity. Mol Neurobiol 2017;54:3783-97.

45. Gomes AP, Price NL, Ling AJ, et al. Declining NAD(+) induces a pseudohypoxic state disrupting nuclearmitochondrial communication during aging. Cell 2013;155:1624-38.

46. Wenz T, Rossi SG, Rotundo RL, et al. Increased muscle PGC-1alpha expression protects from sarcopenia and metabolic disease during aging. Proc Natl Acad Sci U S A 2009;106:20405-10.

47. Wenz T. Mitochondria and PGC-1 $\alpha$ in Aging and AgeAssociated Diseases. J Aging Res 2011;2011:810619.

48. Akhtar S, Siragy HM. Pro-renin receptor suppresses mitochondrial biogenesis and function via AMPK/ SIRT-1/ PGC-1 $\alpha$ pathway in diabetic kidney. PLoS One 2019;14:e225728.

49. Sanz MN, Grimbert L, Moulin M, et al. Inducible Cardiac-Specific Deletion of Sirt1 in Male Mice Reveals Progressive Cardiac Dysfunction and Sensitization of the Heart to Pressure Overload. Int J Mol Sci 2019;20:5005.

(English Language Editor: J. Gray) 